\title{
The Simulation of Viscous Fingering by Diffusion-Limited-Aggregation Model Based on Pressure-Oriented Rule
}

\author{
Wei $\operatorname{Tian}^{1, a^{*}}$, DeTang Lu ${ }^{1, b}$ \\ ${ }^{1}$ Oil and Gas Research Centre, University of Science and Technology of China, Hefei 230026, China

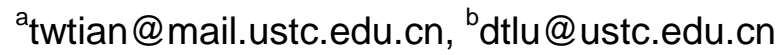

Keywords: Viscous fingering; DLA; 3D digital core; LBM.

Abstract. Viscous fingering phenomenon happens during $\mathrm{CO} 2$ flooding because of viscosity differences between $\mathrm{CO} 2$ and crude oil. It leads to premature breakthrough of displacement fluid and decrease of sweep efficiency. In this paper, a three dimensional Diffusion-Limited-Aggregation model based on pressure-oriented rule is presented to account for the effect of viscosity ratio, injection rate on viscous fingering. Furthermore, Digital cores in our simulation are obtained by CT experiment from real rocks. The results show that viscosity difference is the dominating factor that influences viscous fingering. With the increase of viscosity difference between the two displacement fluids, sweep efficiency decreases dramatically. At appropriate injection rate, the effect of viscous fingering can be decreased.

\section{Introduction}

When a less viscous fluid displaces a more viscous immiscible one, fingering phenomenon happens in the interface [1,2]. As this phenomenon would cause premature breakthrough of displacing fluid and decrease of the sweep efficiency, suppressing the generation of fingering has a significant importance in oil industry. $\mathrm{CO} 2$ as a non-polluting agent has been widely applied in oilfield to displace oil. Under the condition of high temperature and high pressure, $\mathrm{CO} 2$ presents as supercritical state [3], of which the density is close to liquid and the viscosity is close to gas. To investigate viscous fingering in porous media, Paterson [4] simulated viscous fingering in a rectangular plane based on Diffusion-Limited-Aggregation model (DLA) which is creatively proposed by Witten Jr and Sander [5] to simulate diffusion and aggregation of dust suspended in the atmosphere. After that many experts have researched viscous fingering in porous media through experiment and numerical simulation based on DLA model $[6,7,8,9]$. At present, most DLA modeling researches on viscous fingering are two-dimensional and based on traditional DLA model, in which the particle movement process is completely random on the plane. While the actual $\mathrm{CO} 2$ flooding is occurred in three dimensional porous media, and the movement of fluid points is also ruled by pore channel and pressure difference. To investigate these effects and simulate viscous fingering phenomenon more accurately, three-dimensional real digital rocks and the effects of stress field in flow area should be taken into account to improve the traditional DLA model.

\section{Model and Numerical Method}

In traditional DLA model, particles walk completely randomly and the probability in each direction is equiprobable $[4,10]$. Due to heterogeneity of the actual rock formations, the movement of fluid in actual CO2 flooding is also dominated by pore channel and pressure of displacing fluid, the equiprobable rule is not suitable for actual fluid flowing. This paper introduces the regional pressure field and takes the effect of pressure gradient into consideration. The pressure field is calculated by LBM method of D3Q19 model [11,12]. In D3Q19 model, the equilibrium function can be written as:

$$
f_{i}^{e q}=w_{i} \rho\left[1+\frac{c_{i} \cdot u}{c_{s}^{2}}+\frac{\left(c_{i} \cdot u\right)^{2}}{2 c_{s}^{4}}-\frac{u^{2}}{2 c_{s}^{2}}\right]
$$

with 


$$
C=c\left[\begin{array}{ccccccccccccccccccc}
0 & 1 & -1 & 0 & 0 & 0 & 0 & 1 & -1 & 1 & -1 & 1 & -1 & -1 & 1 & 0 & 0 & 0 & 0 \\
0 & 0 & 0 & 1 & -1 & 0 & 0 & 1 & -1 & -1 & 1 & 0 & 0 & 0 & 0 & 1 & -1 & 1 & -1 \\
0 & 0 & 0 & 0 & 0 & 1 & -1 & 0 & 0 & 0 & 0 & 1 & -1 & 1 & -1 & 1 & -1 & -1 & 1
\end{array}\right] \quad c_{s}=\frac{c}{\sqrt{3}}, w_{i}=\left\{\begin{array}{c}
1 / 3, c_{i}^{2}=0 \\
1 / 18, c_{i}^{2}=c^{2} \\
1 / 36, c_{i}^{2}=2 c^{2}
\end{array}\right.
$$

Pressure distribution in each grid point can be calculated by LBM microscopic numerical simulation with D3Q19 model in digital cores.

Assuming the pressure at current grid is $\mathrm{P}_{0}$ and at neighboring grid is $\mathrm{P}_{\mathrm{i}}(\mathrm{i}=+\mathrm{x},-\mathrm{x},+\mathrm{y},-\mathrm{y},+\mathrm{z},-\mathrm{z})$. The pressure difference between neighboring and current grid is $\triangle P_{i}$, so the ratio of pressure difference to total pressure difference in corresponding direction can be written as:

$$
\begin{aligned}
& \omega_{i}=\Delta P_{i} / \sum \Delta P_{i} \\
& (i=+x,-x,+y,-y,+z,-z)
\end{aligned}
$$

Considering the randomness of DLA model, movement probability is divided into two parts: reserved probability $\mathrm{P}_{\mathrm{re}}$ and distributed probability $\mathrm{P}_{\mathrm{d}}$. It's obvious that

$$
P_{r e}+P_{d}=1\left(0<P_{r e}<1\right)
$$

if the adjacent pore number around current grid is $\mathrm{N}$, the probability interval length in each direction is

$$
\beta_{i}= \begin{cases}P_{r e} / N+\omega_{i} * \mathrm{P}_{d} & \text { direction } i \text { is pore } \\ 0 & \text { direction } i \text { is solid }\end{cases}
$$

The above is improved DLA model. It considers not only the randomness of viscous fingering, but also the effect of pressure gradient.

\section{Simulation and Discussion}

\section{Sample Pretreatment}

The structure of real porous media is complex and each particle has six directions to advance. Once particles walk to pores with poor connectivity marked by red circle in Fig 1(a), it's difficult for them to escape to other channels. This paper proposed a pretreatment method called Random Motion of Heuristic Particles (RMHP) to investigate connectivity of pores showed in Fig 1(a). The specific process is as follows: put a heuristic particle at the scanned pore and let it walk randomly according to traditional DLA model. If the particle can move to positon around seed particles in limited steps, the current grid has good connectivity and will be marked as effective-pore; if not, the current grid will be marked as dead-pore. Fig 1(b) shows 3D structure comparison before and after pretreatment. It can be seen that the sample after pretreatment has no dead-pores showed in Fig 1(a) and the skeletal structure does not change much.
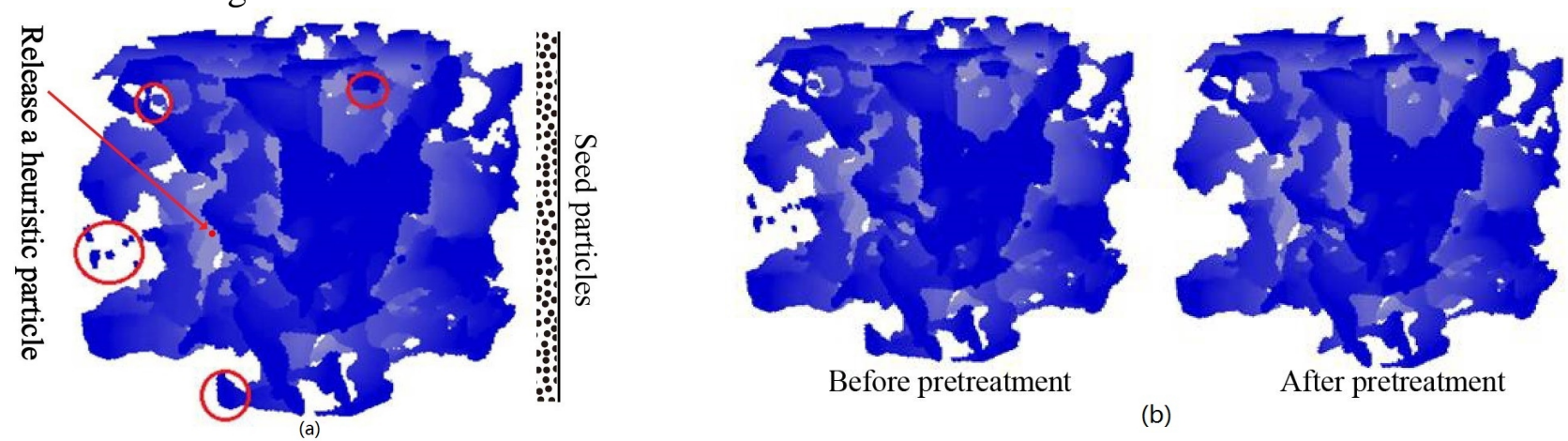

(b)

Fig.1 Sample pretreatment

The sample used in this paper is obtained by CT experiments. The actual size is $4 \mathrm{~mm}^{3}$ and the pixel size of sample image after CT scanning is $100^{3}$. Pixel resolution is $10 \mathrm{um}$ and the porosity is 0.2 . The pressure field in sample after pretreatment is calculated by LBM method [11].

\section{The effect of viscosity}

Under the condition of high temperature and high pressure, $\mathrm{CO} 2$ presents as supercritical state. Its viscosity changes from $20 \mathrm{uPa} . \mathrm{s}$ to $100 \mathrm{uPa}$.s at the temperature range of $20^{\circ} \mathrm{C}$ to $300^{\circ} \mathrm{C}$ and the pressure range of $2 \mathrm{MPa}$ to $80 \mathrm{MPa}$. The viscosity of crude oil is around $1.4 \mathrm{mPa} \cdot \mathrm{s} \sim 1.5 \mathrm{mPa} \cdot \mathrm{s}$. Therefore, 
the range of viscosity ratio is $9 \sim 100$. Here viscosity ratio $\mathrm{M}$ means the ratio of viscosity of crude oil to that of flooding fluid.

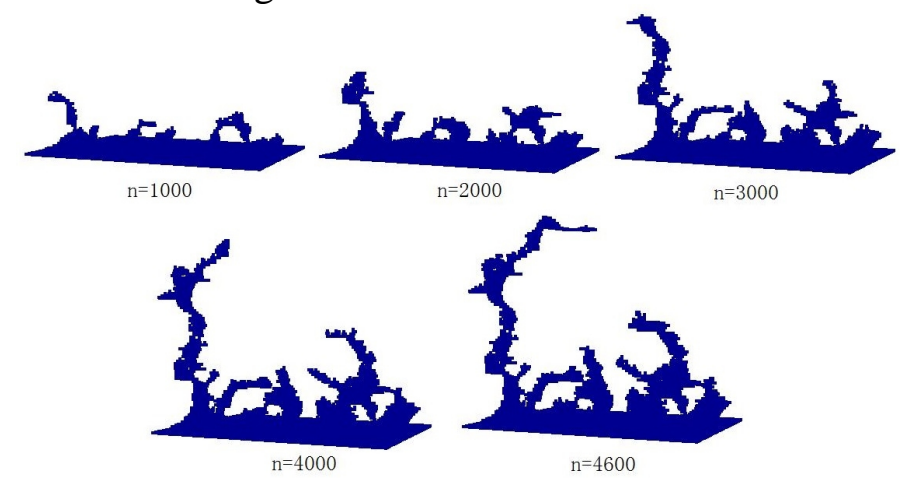

Fig. 2 Injection rate $=2$, viscosity ratio $=1.20$

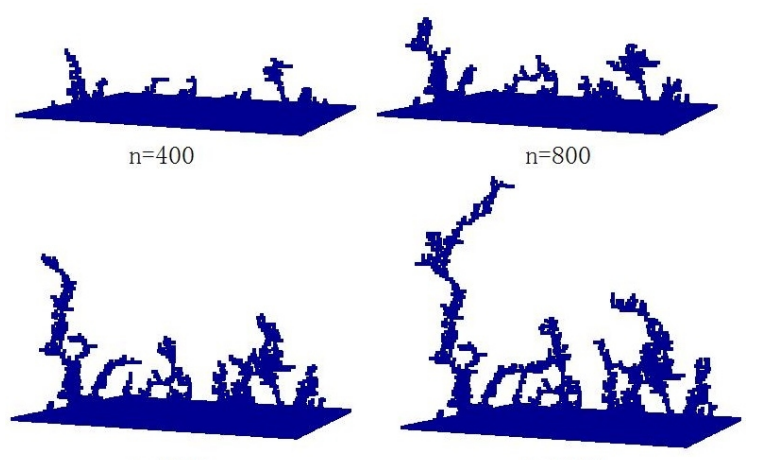

$\mathrm{n}=1200$

$\mathrm{n}=1600$

Fig. 3 Injection rate $=2$, viscosity ratio $=9.00$

The fluid flow channel is showed in Fig. 2 andFig. $3, n$ is the amount of displaced oil. Comparing them, it can be seen that the amount of oil decreases with the increase of viscosity ratio. To describe displacement effect quantitatively, two parameters are introduced here: displacement efficiency $E_{s}$ and fractal dimension $D$. As shown in Fig.4, displacement efficiency and fractal dimension are very large when viscous ratio is close to 1 . With the increase of viscous ratio, displacement efficiency and fractal dimension decrease quickly. When viscous ratio is larger than 20 , both of them change very little, the increase of viscous ratio has little influence on displacement effect.

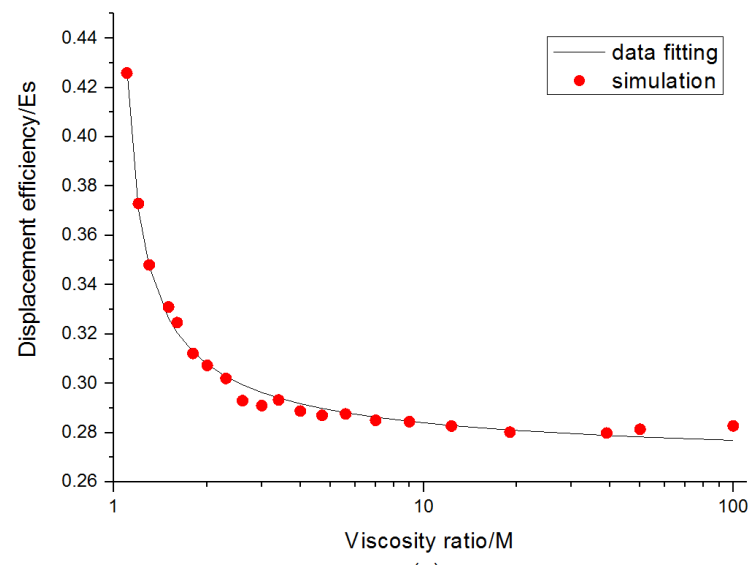

(a)

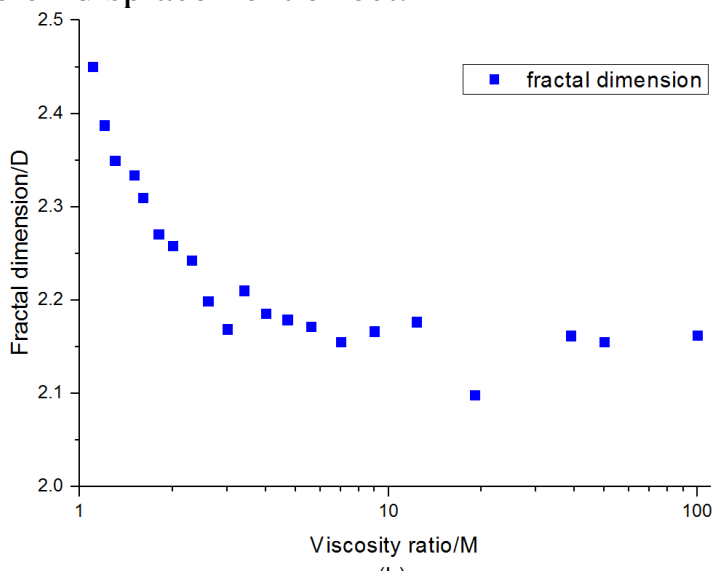

(b)

Fig.4 Relationship between displacement efficiency, fractal dimension and viscosity ratio The effect of injection rate

According to Darcy's Law, small injection rate means small driving pressure. In oil industry, different injection rate has a vital influence on displacement efficiency. One of most probable factors is viscous fingering. Therefore, the effect of injection rate on viscous fingering will be investigated in this section.

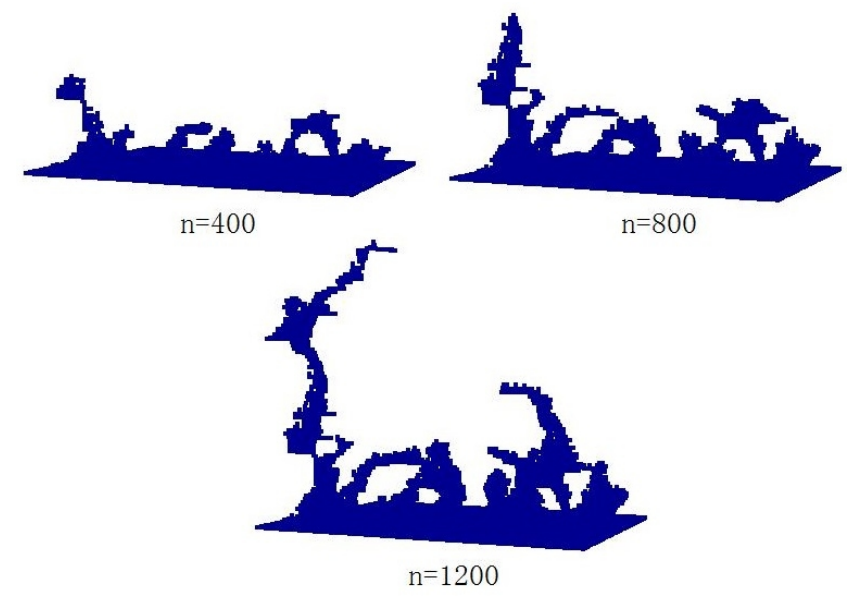

(a)

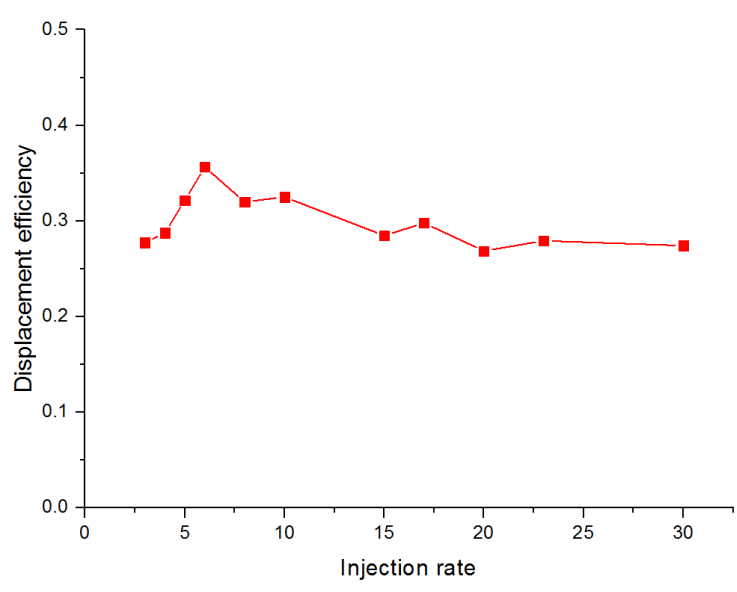

(b)

Fig.5 The effect of injection rate 
Keep viscosity ratio stable and change injection rate, the simulation results are showed in Fig 7. Comparing with Fig 4, it can be seen that the amount of displaced oil decreases with the increase of injection rate under the same viscosity ratio when the front of fingering breaks through the boundary of sample. That is because too large injection rate generates displacing fluid breaks through crude oil along the middle of large pore channel quickly. Later flooding fluid will advance along the channel and displace no more crude oil out from porous media.

The relationship between displacement efficiency and injection rate is showed in Fig 5. It is can be observed that displacement efficiency increases with the increase of injection rate when injection rate is small, but with its continuous rise, displacement efficiency decreases oppositely. That means too large or too small injection rate will reduce displacement effect. Stratum structure in different reservoirs is not the same, threshold pressure and interfacial tension is also various. Only when a suitable injection rate is obtained to suit most pores can we acquire best displacement effect.

\section{Summary}

Viscosity difference is the main factor which leads to viscous fingering during $\mathrm{CO} 2$ flooding. The results show that oil recovery factor decreases rapidly with increase of viscosity when viscosity is less than 20; when viscosity becomes larger, viscous difference has little impact on viscous fingering and displacement efficiency. Thus an important way for improving crude recovery is to decrease viscosity difference between flooding fluid and crude oil.

Injection rate of flooding fluid reflects the size of flooding pressure. Too large flooding rate could result in premature breakthrough of fingering front to form stable flow channel; too low injection rate will make flooding pressure less than starting pressure in some tiny throats and form retention area. Therefore, suitable injection rate should be chosen to achieve best displacement effect.

\section{References}

[1]. Måløy, K. J., J. Feder and T. Jøssang (1985). "Viscous fingering fractals in porous media." Physical review letters 55(24): 2688.

[2]. Saffman, P. (1986). "Viscous fingering in Hele-Shaw cells." Journal of Fluid Mechanics 173: 73-94.

[3]. Cole, D. R., A. A. Chialvo, G. Rother, L. Vlcek and P. T. Cummings (2010). "Supercritical fluid behavior at nanoscale interfaces: Implications for $\mathrm{CO} 2$ sequestration in geologic formations." Philosophical Magazine 90(17-18): 2339-2363.

[4]. Paterson, L. (1984). "Diffusion-limited aggregation and two-fluid displacements in porous media." Physical review letters 52(18): 1621.

[5]. Witten Jr, T. and L. M. Sander (1981). "Diffusion-limited aggregation, a kinetic critical phenomenon." Physical review letters 47(19): 1400.

[6]. Løvoll, G., M. Jankov, K. Måløy, R. Toussaint, J. Schmittbuhl, G. Schäfer and Y. Méheust (2011). "Influence of viscous fingering on dynamic saturation-pressure curves in porous media." Transport in porous media 86(1): 305-324.

[7]. Islam, A., S. Chevalier, I. Ben Salem, Y. Bernabe, R. Juanes and M. Sassi (2014). "Characterization of the crossover from capillary invasion to viscous fingering to fracturing during drainage in a vertical 2D porous medium." International Journal of Multiphase Flow 58: 279-291.

[8]. Herrera-Hernández, E., M. Coronado and H. Hernández-Coronado (2013). "Fractal continuum model for tracer transport in a porous medium." Physical Review E 88(6): 063004.

[9]. Katyal, N., V. Banerjee and S. Puri (2014). "Fractal signatures in analogs of interplanetary dust particles." Journal of Quantitative Spectroscopy and Radiative Transfer.

[10]. Sander, L. M. (1986). "Fractal growth processes." Nature 322(6082): 789-793.

[11]. Qian, Y., D. d'Humières and P. Lallemand (1992). "Lattice BGK models for Navier-Stokes equation." EPL (Europhysics Letters) 17(6): 479. 